\title{
Cross-Sectional Correction for Computing Young's Modulus From Longitudinal Resonance Vibrations of Square and Cylindrical Rods
}

\author{
Wayne E. Tefft and Sam Spinner
}

(December 8, 1961)

\begin{abstract}
The cross-sectional correction involved in the calculation of Young's modulus from the longitudinal resonance vibrations of both square and cylindrical bars has been determined by an empirical method.

On an order of accuracy of 1 part in 1,000, Bancroft's correction, developed for longitudinal waves in cylinders of infinite length was found to be satisfactory. For this purpose the thickness of square bars is related to the diameter of cylindrical bars of the same length by $4 t^{2}=3 d^{2}$.

For accuracies of 1 part in 10,000, modifications in Bancroft's correction must be applied These modifications take a different form for the square rods than for the cylindrical rods. The relation, $2 l=n \lambda$ held for both shapes and on the higher order of accuracy, i.e., the cross-sectional correction was the same for the fundamental and overtones on specimens of the same effective length.
\end{abstract}

\section{Introduction}

This is the fourth in a series of papers $[1,2,3]^{1}$ dealing with the empirical establishment of accurate ${ }^{2}$ relations for computing elastic moduli from the various types of resonance vibrations of isotropic solids. The experimental technique and general approach have been fully described in these previous papers and will not be elaborated here. In one of these papers [2] the problem of the cross-sectional correction factors involved in the computation of Young's modulus from both the fundamental and overtones of the longitudinal resonance vibrations of cylindrical bars has already been treated. The present investigation extends this treatment by considering longitudinal vibrations of square as well as cylindrical bars; and on a higher order of accuracy for both shapes (by a factor of 10) than in the previous work.

Bancroft [4] has obtained an accurate numerical solution of the Pochhammer-Chree equations for the case of longitudinal waves in cylinders of infinite length. However, it is known [5] that these equations cannot be applied rigorously for longitudinal resonance vibrations in bars of finite length (where the ends of the bar are at zero stress). The problem of the present investigation is to determine experimentally the degree to which Bancroft's solution, developed for longitudinal waves in cylinders of infinite length, fits the case for longitudinal resonance vibrations in cylindrical and square bars of finite length; and to what degree modification is required.

\footnotetext{
1 Figures in brackets indicate the literature references at the end of this paper.
} 2 As accurate as the determination of the relevant parameters permit.

\section{Experiment}

Of the eight steel specimens used in this investigation, (table 1) the first six were the same ones used in a previous study [3], and have the same designation. A description of their method of preparation and characterization is given in that reference. This description applies also to the remaining two specimens. The longitudinal modes of vibration were obtained concurrently with the torsional modes discussed in [3]. Consequently, the advantage which was gained in the previous study by cutting down certain larger specimens after a series of resonance frequencies had been determined, and obtaining the resonances on the shortened specimens thus formed (enabling one to obtain a fairly large number of experimental points from a fairly small piece of original stock), is also retained here.

The number of experimental points obtained here, however, was more limited than in the study on torsion because the fundamental and overtones of the longitudinal resonance frequencies of bars are higher than the corresponding torsional ones. It is recalled from the previous paper that the upper frequency limit of the resonance determinations is about $50 \mathrm{kc} / \mathrm{s}$. Consequently, fewer overtones of longitudinal resonances could be obtained before this upper frequency limit was attained; and for the shortest specimen ( $A_{11}$, actually a cube) for which only the fundamental torsional resonance could be determined, not even the fundamental longitudinal resonance frequency could be detected.

The same precautions to insure accuracy, including waiting for the specimens to attain thermal equilibrium with a controlled ambient temperature, that were taken for the torsional resonance determinations, were also used here. The accuracy of these 
resonance determinations, was conservatively estimated to be about 1 part in 10,000, as was the case for torsional resonance.

\section{Results and Analysis}

The frequency of the fundamental and overtones of the longitudinal resonance vibrations of all the specimens is given in table 1.

TABLE 1. Dimensions and longitudinal resonance frequencies of specimens of this investigation

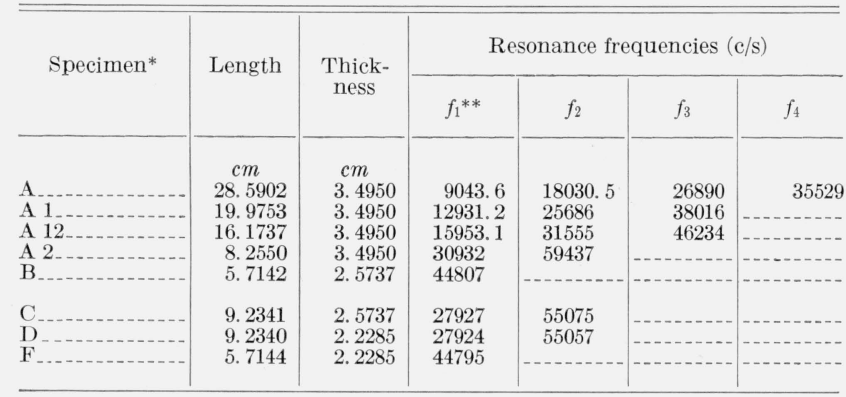

${ }^{*}$ Specimens $\mathrm{B}$ and $\mathrm{C}$ are eylindrical; all others are square in cross section.

$* * f_{1}=$ fundamental resonance frequency, $f_{2}=$ first overtone, etc.

We proceed with an analysis of the data by recalling certain well-known relations, some of which have been used previously [2] in studying this mode of vibration. First, the relation between the velocity, $v_{0}$, of a longitudinal wave in an infinitely thin rod, of infinite length, and the Young's modulus, $E$, and density, $\rho$, of the medium is given by

$$
v_{0}=\sqrt{E / \rho} .
$$

In a cylinder of finite diameter, $d$, and infinite length, the velocity of a longitudinal wave, $v$, is reduced from $v_{0}$. Bancroft's [4] numerical solution of the Pochhammer-Chree equations for this particular case has already been mentioned. His results can be conveniently expressed in the form of a table, such as table 2 , which gives numerical values of the reduction factor,

$$
K_{n}=\left(v_{B} / v_{0}\right)^{2},
$$

as a function of $d / \lambda$ ( $\lambda$ being the wavelength of the wave) and Poisson's ratio, $\mu$, of the medium; $v_{B}$ is the velocity from Bancroft's correction.

For square bars, no theory comparable in accuracy to that developed for cylinders (for this crosssectional correction) appears to be available. However, it seems that the correction factors from table 2 could be applied to square bars if an appropriate assumption were made as to what cross-sectional dimension of a square bar should be taken to correspond to $d$ for a cylinder of the same length. There appears to be some theoretical justification [5] for assuming that the correction would be the same if the polar moments of inertia of the square and circular cross-sectional areas were the same. This assumption was adopted here. Such a condition would require the following relation between the thickness, $t$, of a square bar, and the diameter, $d$, of a cylinder of the same length.

$$
d^{2}=\frac{4}{3} t^{2}
$$

In longitudinal resonance vibrations in cylindrical or square rods (associated with standing waves) the following set of relations are usually adopted,

$$
\lambda=2 l / n, v_{s}=f_{n} \lambda=2 l f_{n} / n
$$

where $l$ is the length of the specimen, $v_{s}$ the "velocity" of the wave, $f$ the longitudinal resonance frequency, and the letter $n$, either as a subscript, or independently, indicates the order of the resonance vibration; for the fundamental, $n=1$; for the first overtone, $n=2$, etc.

Assuming that $v_{B}=v_{s}$, and combining eqs (1), (2), and (4), one obtains the following relation,

$$
E / \rho=\left(1 / K_{n}\right)\left(2 l f_{n} / n\right)^{2} .
$$

Here the subscript in $K_{n}$ takes on the added significance of indicating the order of vibration to which this correction factor applies.

All the parameters in the parentheses in eq (5) may be determined experimentally and $E / \rho$ for a carefully selected group of specimens from the same source should be the same.

The remainder of this paper, then, reduces itself to the problem of determining the degree to which the factor, $K_{n}$, developed for longitudinal waves in cylinders of infinite length also applies to longitudinal resonance vibrations (in rods of finite length). This is equivalent to finding the degree to which the assumption that $v_{B}=v_{s}$ holds. Also to be tested is the assumption that square and cylindrical rods of the same length, having the same polar moments of inertia of cross sectional area, undergo the same reduction in velocity.

In figure 1 , the data of table 1 are plotted on a scale comparable in precision (about 1 part in 1,000) to that of the previous study (fig. 1 of [2]). The square of the parameters comprising the ordinate and abscissa are selected, rather than the first power, as in the previous study, to show the approximately linear relationship that then exists between these variables. Such a presentation is in conformity with table 2 which facilitates accurate interpolation. For the highest accuracy of interpolation from Bancroft's values Aitken's [6] method of interpolation must be used. In order to include square as well as cylindrical specimens, $\beta$, in the figure, is chosen so that $\beta^{2}=(d / 2)^{2}$, or $t^{2} / 3$.

The line in the figure is obtained by plotting Bancroft's values from table 2 for an appropriate value of $\mu$. Such a value may be selected in two independent ways, and the degree to which the values so obtained agree provides a check of the consistency of the data. On the one hand, one may select that value of $\mu$ from table 2 for which the associated values of ordinate and abscissa give the best fit to the experimental data. The value of $\mu$ so obtained was 0.2906 . On the other hand, one may extrapolate 
the experimental values to obtain the best estimate of $v_{0}$; then, knowing $\rho$ for the specimens ${ }^{3}$ from the previous study [3], E may be computed from eq (1). Since $G$ for these specimens is also known from [3], $\mu$ may be computed from the well-known relation, $\mu=(E / 2 G)-1$. The value of $\mu$ so obtained was 0.2880 . This was the value used in the figure. The two values are seen to be in excellent agreement with each other and also with the ones given in the literature for steel.

Examination of figure 1 leads to the following conclusions :

(1) The data of the previous study for cylinders is confirmed in that Bancroft's cross-sectional correction for longitudinal waves in cylinders of infinite length may also be applied to longitudinal resonance vibrations (in cylinders of finite length).

(2) For square specimens, this correction also applies, if the assumption previously made is adopted, namely, that $d^{2}=(4 / 3) t^{2}=4 \beta^{2}$.

The above two conclusions hold only on the order of accuracy of figure 1, i.e., 1 part in 1,000. Small deviations are noted when the data are plotted on a more expanded scale as will be shown in figures 2 and 3.

(3) For both square as well as cylindrical specimens, the observation previously made for cylindrical specimens with respect to overtones still holds. This is that the points for overtones fall on the same line as for fundamental resonance vibrations. This means that the relation, $\lambda=2 l / n$, holds and that the correction factor for the overtones of a longer specimen is the same as for that of a shorter specimen having the same effective length (i.e., having the same value of abscissa).

In figure 2 , the experimental points are plotted on a more extended scale, comparable to the full accuracy of the data itself, namely, about 1 part in 10,000. A convenient way to present such a plot

${ }^{3}$ Actually, since the same set of specimens is involved, $\rho$ should be the same for all specimens and need not be known. Poisson's ratio may then also be computed using $v_{0}$ (longitudinal) and $v_{0}$ (torsional) in place of $E$ and $G$, respectively; $v_{0}$ (torsional) is also known from [3].

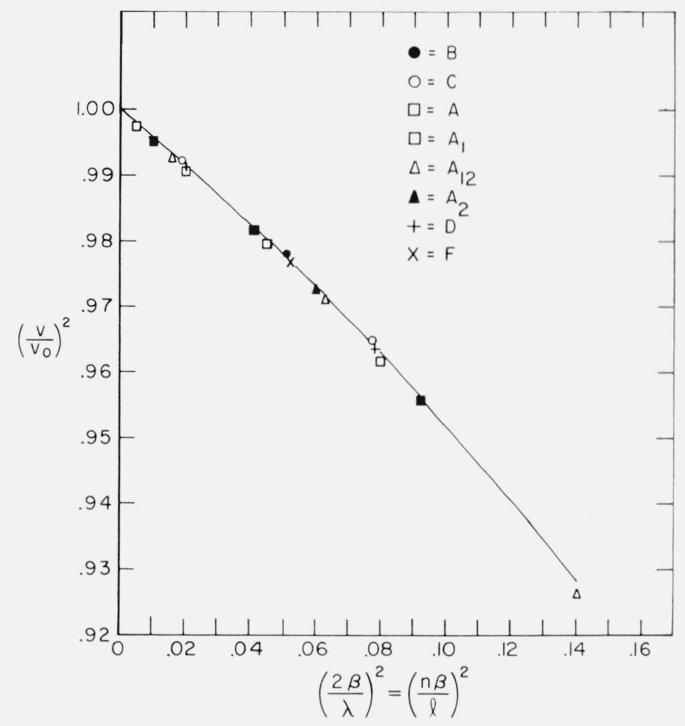

FIGURE 1. Plot showing the reduction in longitudinal velocity, $v$, in a square or cylindrical rod of finite cross section from $v_{0}$, the corresponding velocity in an infinitely thin rod of the same length $l$.

$\beta$ is related to the diameter, $d$, of cylinders, and the thickness, $t$, of square rods by $4 \beta^{2}=d^{2}$ and $3 \beta^{2}=t^{2}$. Also the wavelength, $\lambda=2 l / n$ ( $n$ being the overtone). The line is drawn through Bancroft's values for Poisson's ratio $=0.2880$, showing the agreement between Bancroft's correction, developed for longitudinal waves in cylinders of infinite length, and resonance vibrations in cylinders or square bars of finite length on this scale of accuracy (1 part in 1,000$)$.

is in terms of possible departures of the experimental points from the velocities resulting from Bancroft's correction, $v_{B}$. This would correspond geometrically to making a horizontal line of the one given in figure 1 at $\left(v / v_{B}\right)^{2}=1$, and plotting the ratio $\left(v / v_{B}\right)^{2}$ as a function of $(\beta n / l)^{2}$ for all the experimental points in table 1.

When this procedure is followed, certain significant departures from Bancroft's correction become evident. The upper line shows that the cross-sectional correction factor for cylinders deviates linearly from Bancroft's correction as $(\beta n / l)^{2}$ increases.

TABLE 2. Bancroft's cross-sectional correction factor, $\mathrm{K}_{\mathrm{n}}=\left(v_{\mathrm{B}} / v_{0}\right)^{2}{ }^{\mathrm{a}}$, as a function of Poisson's ratio, $\mu$, and diameter to wavelength ratio, $d / \lambda$

\begin{tabular}{|c|c|c|c|c|c|c|c|c|}
\hline$d / \lambda$ & & .10 & .15 & .20 & .25 & .30 & .35 & .40 \\
\hline 0.00 & 0.0000 & 1. 00000 & 1.00000 & 1.00000 & 1. 00000 & 1. 00000 & 1.00000 & 1. 00000 \\
\hline .05 & .0025 & 0.99988 & 0.99972 & 0.99950 & 0.99922 & 0.99888 & 0.99848 & 0.99802 \\
\hline .10 & .0100 & 99950 & .99886 & .99798 & .99686 & .99549 & .99389 & .99206 \\
\hline .15 & .0225 & .99882 & .99736 & .99533 & .99277 & .98967 & .98609 & .98202 \\
\hline .20 & .0400 & .99780 & .99509 & .99138 & .98670 & .98117 & .97480 & .96772 \\
\hline .25 & .0625 & .99632 & .99184 & .98579 & .97830 & .96956 & .95975 & .94903 \\
\hline .30 & .0900 & .99421 & .98728 & .97810 & .96702 & .95435 & .94049 & .92571 \\
\hline .35 & .1225 & .99114 & .98085 & .96759 & .95203 & .93486 & .91660 & .89770 \\
\hline .40 & .1600 & .98651 & .97158 & .95310 & .93236 & .91031 & .88770 & .86503 \\
\hline .45 & .2025 & .97913 & .95778 & .93300 & .90668 & .88003 & .85372 & .82812 \\
\hline .50 & .2500 & .96621 & .93646 & .90503 & .87383 & .84372 & .81499 & .78780 \\
\hline
\end{tabular}

a $v_{0}$ is the velocity of a longitudinal wave in an infinitely thin cylinder, and $v_{B}$ is the velocity in a cylinder of finite diameter and infinite length. 


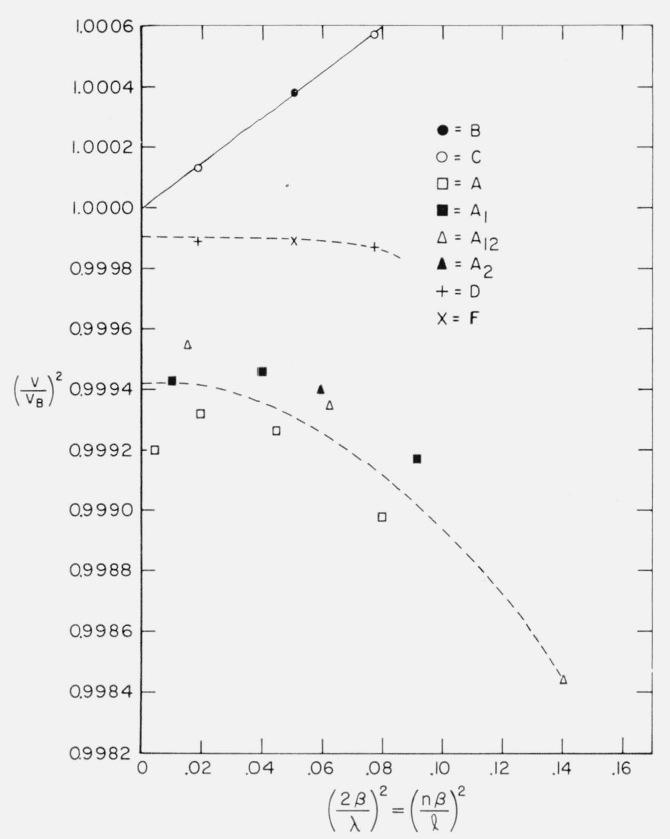

Figure 2. Expanded plot showing departure of longitudinal velocities, associated with resonance vibrations, from values obtained using Bancroft's correction for longitudinal waves, $v_{\mathrm{B}}$, as a function of cross section to length.

Upper line is a least square solution of points for cylindrical specimens while lower two curves are simply drawn through experimental points for smaller and larger square specimens.

This line is obtained by a least squares fit of the experimental points yielding the following relation:

$$
\left(v / v_{B}\right)^{2}=1+0.0075(n d / 2 l)^{2} .
$$

The two lower curves are for the square specimens. The fact that two such curves, one for the larger specimens and one for the smaller ones, appear is disconcerting but presents no serious problem. If the reasonable assumption is made that the separation of these curves is caused by real differences in intrinsic elastic moduli (or $E / \rho$ ) of the large and small specimens due to differences in work hardening or some other cause ${ }^{4}$ and the two curves are brought into coincidence by changing the base value for the lower curve, say, then figure 3 results. Following the procedure of the other figures, the abscissa is again squared in order to obtain an approximately linear relationship. The line in figure 3 is also a least square solution of the experimental points, represented by the equation,

$$
\left(v / v_{B}\right)^{2}=1-0.055\left[1 / 3(n t / l)^{2}\right]^{2} .
$$

It is emphasized that these departures from Bancroft's correction do not mean that this factor is in

\footnotetext{
${ }^{4}$ It is recalled from [3] that the large and small square specimens were cut from 4t is recalled from [3] that the large and small square specimens were cut rom differences only show themselves on the highly expanded scale of figure 2.
}

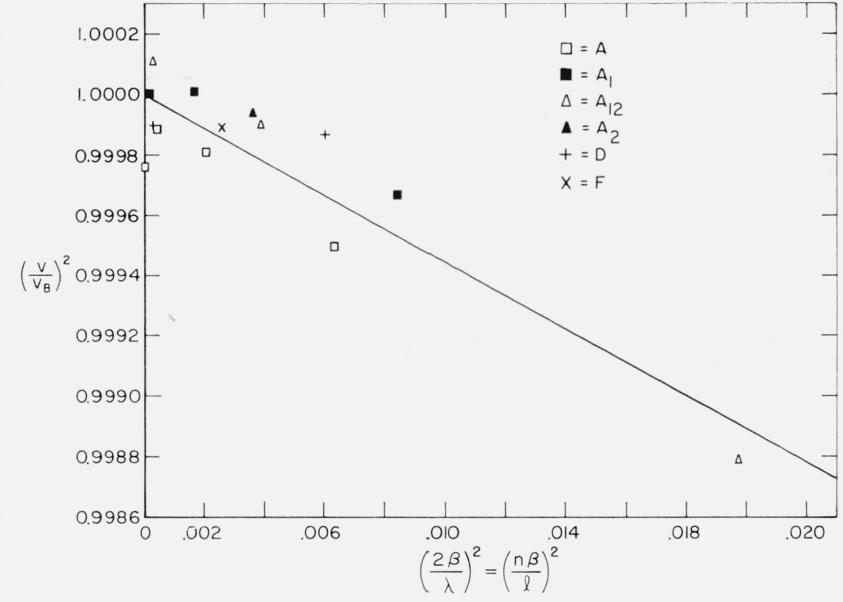

FiguRe 3. Expanded plot showing reduction in longitudinal velocities of square bars, associated with longitudinal resonance vibrations, from that obtained using Bancroft's correction, after adjustment in the base value of the larger specimens.

The line is a least square solution of the experimental points.

error. Rather they are a measure of the degree to which the experimental points depart from Bancroft's values when the conditions of his analysis are not fulfilled.

It is also noted that the third observation on figure 1 concerning the identity of the correction factor for overtones and fundamental resonance vibrations still holds on the expanded scale of figures 2 and 3 . It is recalled from the previous study [3] that this was not the case for torsional resonance vibrations in square rods in which the cross-sectional correction for overtones followed a different pattern from that for the fundamental of specimens of the same effective length. For cylindrical specimens in torsional resonance, on the other hand, no cross-sectional correction at all is required theoretically either for the fundamental or for overtones and this is borne out by experiment.

\section{Summary}

The foregoing analysis may now be summarized in the following way.

In making the cross-sectional correction for longitudinal resonance vibrations of square or cylindrical bars, Bancroft's theoretical correction (developed for longitudinal waves in cylinders of infinite length) may be safely used if accuracy not higher than 1 part in 1,000 is sought. Bancroft's values are presented in such a manner that linear interpolations can also be made to this order of accuracy.

In making a similar correction to an accuracy of 1 part in 10,000, an adjustment in Bancroft's correction factor is required. In computing $E$, from this mode of vibration, then, the following two equations fit the data. 
(a) For cylinders

$$
E=\rho / K_{n}\left(2 l f_{n} / n\right)^{2}\left[1+0.0075(n d / 2 l)^{2}\right] .
$$

(b) For square bars

$$
E=\rho / K_{n}\left(2 l f_{n} / n\right)^{2}\left[1-0.055\left(1 / 3\{n t / l\}^{2}\right)^{2}\right] .
$$

Linear interpolation of Bancroft's values is accurate to 1 part in 10,000 except at the highest values of $d / \lambda=n \beta / l$. In this range Aiken's method of interpolation must be used.

\section{References}

[1] S. Spinner and R. C. Valore, Jr., Comparison of theoretical and empirical relations between the shear modulus and torsional resonance frequencies for bars of rectangular cross section, J. Research NBS 60, 459 (1958) RP2861.

[2] S. Spinner, T. W. Reichard, and W. E. Tefft, A comparison of experimental and theoretical relations between Young's Modulus and the flexural and longitudinal resonance frequencies of uniform bars, J. Research NBS 64A (Phys. and Chem.), No. 2, 147-155 (Mar.-Apr. 1960).

[3] W. E. Tefft and S. Spinner, Torsional resonance vibrations of uniform bars of square cross section, J. Research NBS 65A (Phys. and Chem.), No. 3, 167-171 (MayJune 1961).

[4] D. Bancroft, The velocity of longitudinal waves in cylindrical bars, Phys. Rev. 59, 588-593 (1941).

[5] A. E. H. Love, A treatise on the mathematical theory of elasticity, Fourth Edition, pp. 287-290, Footnote p. 290 (Dover Publications, New York).

(Paper 66A2-153)
[6] A. C. Aitken, Proc. Edinburgh Math. Soc. Ser. 2, 3, 56 (1932). 



\title{
Publications of the National Bureau of Standards ${ }^{*}$
}

\author{
(Including papers in outside journals)
}

\section{Selected Ābstracts}

Tables of spectral-line intensities arranged by wavelengths, W. F. Meggers, C. H. Corliss, and B. F. Scribner, NBS Mono. 32, Pt. II (1961) \$3.00.

The relative intensities, or radiant powers, of 39,000 spectral lines with wavelengths between 2000 and 9000 Angstroms have been determined on a uniform energy scale for seventy chemical elements. This was done by mixing 0.1 atomic percent of each element in powered copper, pressing the powder mixture to form solid electrodes which were burned in a 10 ampere, 220 volt direct-current are, and photographing the spectra with a stigmatic concave grating while a step sector was rotating in front of the slit. The sectored spectrograms facilitated the estimation of intensities of all elements lines relative to copper lines which were then calibrated on an energy scale provided by standardized lamps, and all estimated line intensities were finally adjusted to fit this calibration. Comparisons with other intensity measurements in individual spectra indicate that the National Bureau of Standards spectral-line intensities may have average errors of 20 percent, but first of all they provide uniform quantitative values for the seventy chemical elements commonly determined by spectrochemists. These data are presented by element in part I, and all 39,000 observed lines are given in order of wavelength in part II.

Tables of chemical kinetics. Homogeneous reactions (Supplementary tables), NBS Mono. 34 (Sept. 15, 1961) \$2.75.

A description of these tables and definition of the terms and abbreviations used may be found in the introduction to the 1951 publication. A brief description of the method used in compiling these tables and the limitation of coverage is given in the preface to Supplement 1. A description of the numbering system used in classifying reactions for the tables is given in Supplement 2. The present supplement contains information pertaining to Substitution, Exchange, and Elimination reaction types. It is not complete as still more material falling in these groups is being prepared. The amount of kinetic data to be studied made it seem advisable to present all the material completed to date without further delay. The data on each section give the year and month to which the literature has been surveyed.

Ultraviolet absorption spectrum of ammonia in solid argon at $4.2{ }^{\circ}$ K, K. Dressler, J. Chem. Phys. 35 No. 1, 165-169 (July 1961).

A series of absorption bands in the region $1600 \mathrm{~A}$ to $1900 \mathrm{~A}$ has been observed in thin films of solid argon containing between $0.3 \%$ and $3 \%$ of ammonia. The bands are attributed to isolated $\mathrm{NH}_{3}$, and the missing of alternate bands in the observed vibrational progression shows that in the ground state only the $J=0$ level is appreciably populated. Thermal equilibration of $\mathrm{NH}_{3}$ at $4.2{ }^{\circ} \mathrm{K}$ involves the interconversion of nuclear spin species. It is suggested that the observed rapid equilibration is due to coupling between the proton spins and the spin of the nitrogen nucleus.

Photon-dissociation of water: initial nonequilibrium populations of rotational states of $\mathbf{O H}\left({ }^{2} \mathbf{\Sigma}^{+}\right)$, I. Tanaka, T. Carrington, and H. P. Broida, J. Chem. Phys. 35 No. 2, 750-751 (Aug. 1961).

The irradiation of water vapor by 10 volt photons leads to an excited state which eventually dissociates into a normal hydrogen atom and an electronically excited $\mathrm{OH}$ radical. Our spectroscopic observations of the rotational and vibrational distributions of these $\mathrm{OH}$ radicals show that different excitation energies affect the initial state of the water molecule, and that added inert gases influence the dissociation of this molecule.

Repulsion of energy levels in complex atomic spectra, R. E. Trees, Phys. Rev. 123, No. 4, 1293-1300 (Aug. 15, 1961).

Rosenzweig and Porter have shown a "repulsion of energy levels" in spacing distributions determined from energy levels in complex atomic spectra. The present paper extends their work by showing that these spacing distributions can be determined from calculated positions of the levels in these spectra. Since calculated data are available for spectra where the observed data are scarce or incomplete, this partially overcomes limitations imposed by statistical inaccuracy when direct use is made of the observed data. The equivalence of the two approaches is demonstrated by showing that calculated data for $\mathrm{Ta}$ II yield the same spacing distribution as obtained from observed data for Ta II and Re I combined. These are complex spectra in which a fully developed repulsion effect is present. A similar demonstration of equivalence is carried out for spectra of Ru I and Mo I, where the repulsion effect is in an intermediate state of development. The results also indicate that numbers easily evaluated from the radial parameters of the theory will indicate roughly the degree of repulsion, replacing to some extent the need for an explicit calculation of the spacing distribution.

Infrared spectrum and structures of the $\mathrm{NF}_{2}$ radical, $\mathrm{M}$. D. Harmony, R. J. Myers, L. J. Schoen, D. R. Lide, Jr., and D. E. Mann, J. Chem. Phys. 35, No. 3, 1129 (Sept. 1961). The infrared spectrum of the $\mathrm{NF}_{2}$ radical has been detected in a heated sample of $\mathrm{N}_{2} \mathrm{~F}_{4}$. The stretching vibrations $\mathrm{v}_{1}$ and $\mathrm{v}_{3}$ are assigned as $1074 \mathrm{~cm}^{-1}$ and $935 \mathrm{~cm}^{-1}$, respectively. The former band shows well-resolved rotational structure, from which one obtains $A-(B+C) / 2=1.93+0.01 \mathrm{~cm}^{-1}$. This constant implies an FNF angle of about $104^{\circ}$.

Statistical dynamics of simple cubic lattices. Model for the study of Brownian motion II, R. J. Rubin, J. Math. Phys. 21, No. 3, 373-386 (May-June 1961).

New results concerning the statistical dynamics of a heavy particle in an $n$-dimensional $(n \mathrm{D})$ cubic lattice are presented. In a well-defined sense, the random motions of a heavy particle in a $1 \mathrm{D}$ lattice and a $3 \mathrm{D}$ lattice are accurately described by Kramers' equation for a free particle and a harmonically bound particle, respectively. A related, but not independent, resalt, is that the velocity $v(t)$ and position $u(t)$ of a heavy particle in a $1 \mathrm{D}$ lattice and a $3 \mathrm{D}$ lattice constitute two dimensional stationary gaussian Markoff processes. It is definitely established that in the case of a $2 \mathrm{D}$ lattice the stationary gaussian process $\left(\begin{array}{l}v(t) \\ u(t)\end{array}\right)$ is non-Markoffian. In the course of the analysis, several interesting connections between solutions of the discrete lattice equations of motion and solutions of the corresponding continuum equation of motion (the $n \mathrm{D}$ wave equation) are uncovered.

Mass spectrometric study of the thermal dissociation of $\mathrm{N}_{2} \mathrm{~F}_{4}$, J. T. Herron and V. H. Dibeler, J. Chem. Phys. 35, No. 2 , $747-748$ (Aug. 1961).

The bond dissociation energy $\mathrm{D}\left(\mathrm{F}_{2} \mathrm{~N}-\mathrm{NF}_{2}\right)$ has been measured by a mass spectrometric method to be $21.5 \mathrm{kcal} / \mathrm{mole}$. This is considered accurate within $1.6 \mathrm{kcal} / \mathrm{mole}$.

Effect of branching on the thermal decomposition of polymers, L. A. Wall, Soc. Chem. Ind. Mono. 13, pp. 146-162 (Page Bros. (Norwich) Ltd., England, 1961). 
The rates of volatilization for certain linear polymers, for example, polyethylene and polypropylene and copolymers of ethylene and propylene, prepared using Ziegler-type catalyst, show maxima in the region of 20 to $40 \%$ volatilization, or conversion. On the other hand, polymers prepared from the same monomers under conditions that produce branched molecules show a continuous decrease in the rate of volatilization with conversion. The presence of a maximum indicates the operation, at least partially, of an overall random mechanism of scission. Theoretical treatments have previously been made by Simha on the random breakdown of branched chains and on the random breakdown perturbed by allowing chain bonds at branched points to rupture more rapidly than the other chain bonds. The net result was that branched chains per se do not account for the lack of maxima in the rate curves, although experimentally the influence of the branches is apparent. From a study of degradation rates of approximately forty polymers having varying numbers and lengths of branches, the results show that in paraffinic hydrocarbon polymers containing three or more methyl end groups per 100 methylene chain groups, the maxima are eliminated provided the branches contained two or more carbon atoms.

Phase equilibria research in systems involving the rare earth oxides, R. S. Roth, Book, Rare Earth Research Pt. II, pp. 88-95 (The MacMillan Company, New York, N.Y., 1961).

This manuscript is a review of the unpublished phase equilibria research at present being conducted at the National Bureau of Standards on systems involving rare earth oxides with: (1) oxides of other trivalent ions, (2) $\mathrm{Nb}_{2} \mathrm{O}_{5}$ or $\mathrm{Ta}_{2} \mathrm{O}_{5}$, and (3) $\mathrm{B}_{2} \mathrm{O}_{3}$. Simplified solid-state phase diagrams are shown for the systems involving oxides of trivalent rare earth ions and $\mathrm{Al}_{2} \mathrm{O}_{3}, \mathrm{Ga}_{2} \mathrm{O}_{3}, \mathrm{Cr}_{2} \mathrm{O}_{3}, \mathrm{Fe}_{2} \mathrm{O}_{3}, \mathrm{Se}_{2} \mathrm{O}_{3}$ and $\mathrm{In}_{2} \mathrm{O}_{3}$.

Degradation of poly (2,3,4,5,6-pentafluorostyrene), L. A. Wall, J. M. Anotnucci, S. Straus, and M. Tyron, Soc. Chem. Ind. Mono. No. 13, 295-302 (1961).

The rates of thermal decomposition of a ploymer of 2,3 , 4,5,6-pentafluorostyrene have been measured, and from these data an activation energy value of 65 kcal per mole was calculated. A qualitative evaluation was also made of the behavior of the polymer in photo-oxidation. The results of both studies indicate that the material is to a surprising degree more stable than polystyrene. Although further mechanism studies are necessary for a complete understanding of the effects observed, it seems evident that the alpha $\mathrm{C}-\mathrm{H}$ bonds and the $\mathrm{C}-\mathrm{C}$ bonds of the polymer chain are stronger in the poly, pentafluorostyrene. This is interpreted to be the result of decreased resonance interaction between the chain bonds and the phenyl groups due to the presence of fluorine atoms on the phenyl ring.

The half-life of carbon-14, W. B. Mann, W. F. Marlow, and E. E. Hughes, Intern. J. Appl. Radiation and Isotopes 11, No. 2, 57-67 (1961).

The half-life of carbon-14 has been determined with an accuracy believed to be higher than that of previously reported values. Carbon dioxide was liberated by the action of perchloric acid on barium carbonate which contained $\mathrm{C}^{14}$ in approximately 44 percent isotopic abundance. Samples of this $\mathrm{C}^{14}$ dioxide were analyzed mass spectrometrically, and other portions were diluted quantitatively with inert carbon dioxide and counted in the NBS length-compensated internal gas counters. Technical difficulties encountered in this work, which are characteristic of this type of experiment, particularly problems of adsorption and the methods used to overcome them, are discussed in detail. On the basis of a thorough analysis of all results obtained, the half-life of $\mathrm{C}^{14}$ is believed to be $5760 \pm 50$ years, where the indicated uncertainty denotes an estimated overall probable error of the result.

Rate of the reaction $\mathrm{NO}+\mathrm{N}, \mathrm{J}$. T. Herron, J. Chem. Phys. 35, No. 3, 1138-1139 (Sept. 1961).

The rate of the reaction $\mathrm{NO}+\mathrm{N}$ has been measured to be $k=1.0 \pm 0.5 \times 10^{13} \mathrm{cc} . \mathrm{mole}^{-1} \mathrm{sec}^{-1}$.
Transition probabilities in multilevel system: calculation from impulsive and steady-state experiments, T. Carrington, J. Chem. Phys. 35, No. 3, 807-816 (Sept. 1961).

The study of collison induced transitions of molecules in a system of many energy levels is discussed in an analysis of time dependent and steady state experiments. These results are used in a discussion, with numerical examples, of the difficulties in calculating the transition probabilities from observed population distributions.

An intimate conceptual and mathematical relationship is established between two types of experiments which are possible at present. One is an impulse, or time dependent experiment such as excitation with a fast flash lamp and snapshot observation after a known delay time. The other is a steady state experiment in which molecules are fed into the system (a gas or a surface) at a single energy level, for example, and undergo transitions among the available levels in competition with a first order removal process such as spontaneous radiation or description from a surface.

The ideas summarized above are used to develop procedures for calculating transition probabilities from observed population distributions. Numerical examples and qualitative considerations indicate that when transitions involving more than single quantum jumps are involved, the experimental data must be known to much higher accuracy than that desired in the transition probabilities. The difficulties increase rapidly as the number of levels in the system increases.

Perturbations and rotational intensities observed in $\mathrm{CN}$ bands emitted by reactions of organic molecules with nitrogen atoms, N. H. Kiess and $\mathrm{H}$. P. Broida, J. Mole. Spect. 7, No. 3, 194-208 (Sept. 1961).

The rotational perturbations between the $v=0$ level of the $\mathrm{B}^{2} \Sigma^{+}$electronic state and the $v=10$ level of the $A^{2} \pi_{i}$ electronic state of $\mathrm{CN}$ have been re-investigated. Rotational analyses of the 10.3 and 10.5 bands of the $A^{2} \pi-X^{2} \Sigma$ system and of the 0.0 band of the $B^{2} \Sigma-X^{2} \Sigma$ system have been made. The relative intensities of the perturbed lines, extra lines, and neighboring unperturbed lines have been studied as a function of pressure from 0.1 to $100 \mathrm{~mm} \mathrm{Hg}$. A new pair of extra lines has been found. At the higher pressures the populations of the excited rotational levels of $\mathrm{CN}$ approach a Boltzmann distribution. As the pressure is lowered the intensities of the perturbed and extra lines become continually more anomalous. The interpretation is given that $\mathrm{CN}$ is formed in the $A^{2} \pi$ state more than 20 times as readily as it is formed in the $B^{2} \Sigma$ state in reactions of organic molecules with the nitrogen afterglow.

Molecular structure of propylene, D. R. Lide and D. Christensen, J. Chem. Phys. 35, No. 4, $1374-78$ (Oct. 1961).

The microwave spectra of seven isotopic species of propylene have been studied in order to obtain an accurate molecular structure. The complete $r_{s}$ (substitution) structure has been calculated. The more important parameters are: $r(\mathrm{C}-$ $\mathrm{C})=1.336+0.004 \mathrm{~A}, r(\mathrm{C}-\mathrm{C})=1.501+0.004 \quad \mathrm{~A}, \quad \Varangle \mathrm{CCC}=$ $124.3^{\circ} \pm 0.3^{\circ}$. The structure is compared with those of related molecules. It is concluded that no difference can be detected in the double-bond lengths in ethylene, propylene, and the vinyl halides. The $\mathrm{CC}$ single-bond length in propylene is indistinguishable from that in acetaldehyde and other acetyl compounds, and is $0.025 \mathrm{~A}$ shorter than the $\mathrm{CC}$ distance in saturated hydrocarbons. In the $-\mathrm{CH}_{2}$ group in propylene the $\mathrm{CH}$ bond trans to the methyl group appears slightly shorter than the cis CH bond; a similar effect occurs in the vinyl halides.

Low-angle X-ray diffraction of crystalline nonoriented polyethylene and its relation to crystallization mechanisms, L. Mandelkern, A. S. Posner, A. F. Diorio, and D. E. Roberts, J. Appl. Phys. 32, No. 8, 1509-1517 (Aug. 1961).

X-ray diffraction maxima at low angles have been observed in crystalline but non-oriented linear polyethylene, the crystallization process being conducted from the melt of the pure undiluted polymer. Several orders of diffraction are observed in favorable cases, and the spacings corresponding 
to the first-order reflections range from 150 to $850 \mathrm{~A}$. The values of the maxima depend on the mode of crystallization. Previous assertions that in such systems the maxima are limited to $100-200 \mathrm{~A}$ are shown to be overly restrictive and typical only of erystallization processes conducted at very large undercoolings.

Major attention is focused on the properties of specimens crystallized isothermally at relatively low values of the undercooling. The spacings are very sensitive to the crystallization temperature in this range. The highest values are observed at low undercooling and substantial decreases occur as the temperature is lowered. Concomitantly, the density observed after isothermal crystallization significantly decreases with a lowering of the crystallization temperature.

The fact that a periodicity can be developed in such systems, the magnitude of the maxima, and their dependence on the crystallization temperature, is explicable by the application of nucleation theory. It is assumed that subsequent to the formation of critical-size nuclei from a bundle of polymer chains, crystal growth along the chain direction is severely retarded, while in the transverse direction essentially unimpeded crystallization occurs. From the observed temperature coefficient of the low angle spacings the ratio of the excess free energy (due to the junction of crystalline and amorphous regions at the crystallite ends) to the bulk enthalpy of fusion is found to be 2.6. The magnitude of this ratio receives confirmation from other types of experiments.

Intramolecular rearrangements. II. Photolysis and radiolysis of 4-methyl-2-hexanone, P. J. Ausloss, J. Phys. Chem. 65, 1616-1618 (1961).

In the photolysis and radiolysis of 4-methyl-2-hexanone, three butene isomers are formed by an intramolecular rearrangement process in which a secondary or primary $\gamma$ hydrogen is transferred to the carbonyl group.

In the vapor-phase photochemical decomposition, the ratios 1-butene/2-butene and cis-2-butene/trans-2-butene increase with decrease in wavelength and increase in temperature. In the liquid-phase photolysis, however, no dependence on wavelength was observed, although the effect of temperature on the butene distribution was very pronounced. Similar results were obtained in the photochemical decomposition of sec-butyl acetate. In the transition of the liquid to the solid phase there was a drastic change in the butene distribution. The butene distribution obtained in the photosensitized decomposition at $2537 \mathrm{~A}$ agrees closely with the one obtained in the nonsensitized decomposition at the same wavelength. A comparison of the results obtained in the photolysis with those obtained in the radiolysis in the vapor and liquid phase indicates that in the latter case the butenes may be formed by way of a highly electronically excited molecule.

Infrared spectra of carbon monoxide as a solid and in solid matrices, A. G. Maki, J. Chem. Phys. 35, No. 3, 931-935 (Sept. 1961).

The fundamental infrared absorption frequency of several isotopes of $\mathrm{CO}$ has been observed in solid carbon monoxide and in the solid matrices $\mathrm{N}_{2}, \mathrm{Ar}$, and $\mathrm{CH}_{4}$. In addition, the overtone in solid carbon monoxide has been measured. It is shown that frequency shifts due to isotopic substitution in the solid state follow the formulas developed for the gas phase only when molecules of the same isotopic species are not adjacent to each other, i.e., only in dilute solutions.

\section{Other NBS Publications}

Journal of Research 66B (Math. and Math. Phys.) No. 1 (Jan.-Mar. 1962) 75 cents.

Error bounds for eigenvectors of self-adjoint operators N. W. Bazley and D. W. Fox.

Intermediary equatorial orbits of an artificial satellite. J. P. Vinti.

Selected bibliography of statistical literature 1930 to 1957: V. Frequency functions, moments, and graduation. L. S. Deming.

Measurement of wave fronts without a reference standard: Part 2. The wave front-reversing interferometer. J. B. Saunders.
Journal of Research 66C (Eng. and Instr.) No. 1 (Jan.-Mar. 1962) 75 cents.

Reference tables for 40 percent iridium-60 percent rhodium versus iridium thermocouples. G. F. Blackburn and F. R. Caldwell.

A method for the self-calibration of attenuation-measuring systems. R. L. Peck.

Special shielded resistor for high-voltage d-c measurements. J. H. Parks.

Voltage ratio measurements with a transformer capacitance bridge. T. L. Zapf.

Weight calibration schemes for two-knife-edge direct-reading balances. H. E. Almer, L. B. Macurdy, H. S. Peiser, and E. A. Weck.

Tunnel diode large-signal equivalent circuit study and the solutions of its nonlinear differential equations. S. B. Geller and P. A. Mantek.

A missile technique for the study of detonation waves. F. W. Ruegg and W. W. Dorsey.

Creep of cold-drawn nickel, copper, 70 percent nickel-30 percent copper, and 30 percent nickel-70 percent copper alloys. W. D. Jenkins and W. A. Willard.

Journal of Research 66D (Radio Prop.) No. 2 (Mar.-Apr. 1962) 70 cents.

Atmospheric phenomena, energetic electrons, and the geomagnetic field. J. R. Winckler.

The summer intensity variations of [OI] $6300 \mathrm{~A}$ in the tropics. D. Barbier, F. E. Roach, and W. R. Steiger.

Generation of radio noise in the vicinity of the earth. P. A. Sturrock.

Fading characteristics observed on a high-frequency auroral radio path. J. W. Koch and $H$. E. Petrie.

Some problems connected with Rayleigh distributions. M. M. Siddiqui.

Impedance of a monopole antenna with a radial-wire ground system on an imperfectly conducting half space, Part I. S. W. Maley and R. J. King.

Theory of the infinite cylindrical antenna including the feedpoint singularity in antenna current. R. H. Duncan.

The $E$-field and $\dot{H}$-field losses around antennas with a radial ground wire system. T. Larsen.

The electric field at the ground plane near a disk-loaded monopole. J. Hansen and T. Larsen.

An experimental study of phase variations in line-of-sight microwave transmissions, K. A. Norton, J. W. Herbstreit, H. B. Janes, K. O. Hornberg, C. F. Peterson, A. F. Barghausen, W. E. Johnson, P. I. Wells, M. C. Thompson, Jr., M. J. Vetter, and A. W. Kirkpatrick, NBS Mono. 33 (Nov. 1, 1961) 55 cents.

Bibliography and index on vacuum and low pressure measurement, W. G. Brombacher, NBS Mono. 35 (Nov. 10, 1961) 60 cents.

Effect of mortar properties on strength of masonry, C. C. Fishburn, NBS Mono. 36 (Nov. 20, 1961) 30 cents.

Tabulation of data on microwave tubes, C. P. Marsden, W. J. Keery, and J. K. Moffitt, NBS Handb. 70, (Nov. 1, 1961) $\$ 1.00$.

Safety rules for the installation and maintenance of electric supply and communications lines. Comprising Part 2, the definitions, and the grounding rules of the sixth edition of the National Electrical Safety Code, NBS Handb. 81, (1961) $\$ 1.75$

Mean electron density variations of the quiet ionosphere, No. 6-August 1959, J. W. Wright, L. R. Wescott, and D. J. Brown, NBS Tech. Note 40-6 (PB151399-6) (1961) $\$ 1.50$.

Techniques for computing refraction of radio waves in the troposphere, E. J. Dutton and G. D. Thayer, NBS Tech. Note 97 (PB161598) (1961) \$1.50.

Performance predictions for single tropospheric communication links and for several links in tandem, A. P. Barsis, K. A. Norton, P. L. Rice, and P. H. Elder, NBS Tech. Note 102 (PB161603) (1961) \$3.00.

Mode calculations for VLF propagation in the earth-ionosphere waveguide, K. P. Spies and J. R. Wait, NBS Tech. Note 114 (PB161615) (1961) \$1.50. 
Astrophysical and plasma physics research at the National Bureau of Standards, Highlights for 1961, L. M. Branscomb, K. E. Shuler, J. A. Suddeth, NBS Tech. Note 116 (PB161617) (1961) \$1.00.

Variations in frequency of occurrence of sporadic $E$ 19491959, W. B. Chadwick, NBS Tech. Note 117 (PB161618) (1961) 75 cents.

Electrical conduction in $p$-type titanium sesquioxide, J. Yahia and H. P. R. Frederikse, Phys. Rev. 123, No. 4, 1257-1261 (Aug. 15, 1961).

Parameters $\alpha$ and $\beta$ in the spectra of the iron group. R. E. Trees and C. K. Jorgensen, Phys. Rev. 132, No. 4, 12781280 (Aug. 15, 1961).

Slow drift solar radio bursts: harmonic frequency ratios, solar longitude dependence, and frequency drift rates, M. B. Wood, Australian J. Phys. 14, No. 2, 234-241 (1961).

Fibrous silica, W. Haller, Nature 191, No. 4789, 662-663 (Aug. 12, 1961).

Control terminology-A report on U.S. standards activity H. L. Mason, Control Eng. 8, No. 10, 67-70 (Oct. 1961).

Behavior and evaluation of rubber, R. D. Stiehler, Am. Concrete Pipe Assoc. Tech. Memo. (Oct. 1961).

Partial confounding in fractional replication, W. J. Youden, Technometric 3, No. 3, 353-358 (Aug. 1961).

Welded butt joints with fine wires, L. Martz, Rev. Sci. Instr., 32, No. 8, 990-991 (Aug. 1961).

Systematic errors in physical constants, W. J. Youden, Physics Today 14, No. 9, 32-34, 36, 38, 40, 42 (Sept. 1961).

A study of solar activity associated with polar cap absorption (abstract), C. S. Warwick and M. B. Wood, Polar Cap Absorption Conf. (Kiruna, Sweden, Aug. 8-11, 1960), Arkiv Géofyski 3, No. 21, 457 (1960).

$\mathrm{Kr}^{86}$ and atomic-beam-emitted $\mathrm{Hg}^{198}$ wavelengths, R. L. Barger and K. G. Kessler, J. Opt. Soc. Am. 51, No. 8, 827829 (Aug. 1961).

Excess noise in microwave detector diodes, J. J. Faris and J. M. Richardson, IRE Trans. Microwave Theory and Tech. MTT-9, No. 4, 312-314 (July 1961).

Conductance of solutions of water, acetic anhydride, and acetyl chloride in acetic acid, T. B. Hoover and A. W. Hutchinson, J. Am. Chem. Soc. 83, No. 16, 3400-3405 (1961).

Amine buffers for $p \mathrm{H}$ control, R. G. Bates, Ann. N.Y. Acad. Sci. 92, No. 2, 341-356 (June 1961).

Exact and approximate distributions for the Wilcoxon statistic with ties, S. Y. Lehman, J. Am. Stat. Assoc. 56, 293298 (June 1961).

Some geometrical theorems for abscissas and weights of gauss type, P. J. Davis and P. Rabinowitz, J. Math. Anal. Appl. 2, No. 3, 428-437 (June 1961).

Some relationships between short-wave fadeouts, magnetic crochets, and solar flares, L. W. Acton, J. Geophys. Research 66, 3060-3063 (Sept. 1961).

A simple calibration technique for vibrating sample and coil magnetometers, N. V. Frederick, Proc. IRE 49, 1449 (Sept. 1961).

First pulsed radio soundings of the topside of the ionosphere, R. W. Knecht, T. E. Van Zandt, and S. Russell, J. Geophys. Research 66, 3078-3081 (Sept. 1961).

Performance characteristics of turbine flowmeters, M. R. Shafer, Trans. ASME J. Basic Eng. Paper No. 61-W A-25 (1961).
Periodicity modulo $m$ and diversibility properties of the partition function, M. Newman, Trans. Am. Math. Soc. 97, No. 2, 225-236 (Nov. 1960).

The synthesis of food, A. T. McPherson, Ind. Research, pp. 20-27 (Nov. 1961).

Effect of mercury-alloy ratio on the physical properties of amalgams, W. T. Sweeney and C. L. Burns, J. Am. Dental Assoc. 63, No. 9, 374-381 (Sept. 1961).

Measurement characteristics of farm milk tanks, M. W. Jensen, Scale J., pp. 4-5 (Oct. 1961).

Applications of statistics in Post Office automation, B. M. Levin and N. C. Severo, Am. Statistician 15, No. 4, 14-18 (Oct. 1961)

Experimental design and the ASTM committees, W. J. Youden, Materials Research and Standards, 862-867 (Nov. 1961)

Present status of panoramic roentgenography, J. W. Kumpula, J. Am. Dental Assoc. 63, No. 8, 194-200 (Aug. 1961).

Sun-time replaced by atomic clocks, R. S. Tipson, Capital Chemist 11, 255 (Nov. 1961).

What is the best value? W. J. Youden, J. Wash. Acad. Sci. 51, No. 6, 95-97 (Oct. 1961).

The permanent function as an innerproduct, M. Marcus and M. Newman, Bull. Am. Math. Soc. 67, No. 2, 223-224 (March 1961).

Thermal conductivity of some commercial iron-nickel alloys, T. W. Watson and H. E. Robinson, Trans. ASME, Series C, J. Heat Transfer 83, No. 4, 403-408 (Nov. 1961).

Exploratory research on demineralization, A. Rose, R. F. Sweeny, T. B. Hoover, V. N. Schrodt, Ch. in Book, Saline Water Conversion, Advances in Chemistry Series No. 27, 50-55 (American Chemical Society, Washington, D.C., 1960).

Measurement of the transition probability of the OI multiplet at $6157 \mathrm{~A}$, W. L. Wiese and J. B. Shumaker, Jr., J. Opt. Soc. Am. 51, No. 9, 937-942 (Sept. 1961).

Deposition of metals from the vapor phase and similarity of the process to electrodeposition, A. Brenner, Trans. Inst. Metal Finishing 38, No. 4, 123-130 (Aug. 1961).

Photographic response to successive exposures of different types of radiation, M. Ehrlich and W. L. MeLaughlin, J. Opt. Soc. Am. 51, No. 11, 1172-1181 (Nov. 1961).

On the possibility of rejecting certain modes in VLF Propagation, J. R. Wait, Proc. IRE, Letter 49, 1429-1430 (Sept. 1961).

The relaxation times of some paramagnetic dispersions, P. H. Fang, Physica 27, 68 (1961).

Multiple biologic recording for digital analysis, H. L. Mason, Proc. Interdisciplinary Clinic on the Instrumentation Requirements for Psychophysiological Research, FIER Clinic on Psychophysiological Instrumentation, Lafayette Clinic, pp. 65-67 (May 16-17, 1961).

* Publications for which a price is indicated (except for Technical Notes) are available only from the Superintendent of Documents, U.S. Government Printing Office, Washington 25, D.C. (foreign postage, one-fourth additional). Technical Notes are available only from the Office of Technical Services, U.S. Department of Commerce, Washington 25, D.C. (Order by PB number.) Reprints from outside journals and the NBS Journal of Research may often be obtained directly from the authors. 